\title{
Evaluation of patients admitted with musculoskeletal tuberculosis: sixteen years' experience from single center in Turkey
}

Mikail Ozdemir

Osmaniye Tuberculosis Dispensary

Hanife Gulnihal Ozdemir ( $\sim$ dr.gulnihalzdmr@gmail.com )

Osmaniye State Hospital

\section{Research Article}

Keywords: bursitis, extrapulmonary, Pott's spine, tuberculosis dispensary

Posted Date: February 23rd, 2021

DOl: https://doi.org/10.21203/rs.3.rs-224407/v1

License: (c) (1) This work is licensed under a Creative Commons Attribution 4.0 International License.

Read Full License 


\section{Abstract \\ Background}

The aim of our study was to elaborate musculoskeletal system TB (tuberculosis) as a single-center experience.

\section{Methods}

This is a retrospective observational study conducted at a TB Dispensary in the east Mediterranean part of Turkey between 2004 and 2020. The clinical and demographic characteristics including age, gender, involvement location and duration of illness, presenting complaint, local examination findings, treatment outcome were retrieved and analyzed from the case files. Statistical analyses were performed using SPSS Statistics version 17.0 (IBM). The normality of data analysed by using Kolmogorov-Smirnov. The descriptive statistics were reported as mean \pm standard deviation, medians, and ranges (min-max).

\section{Results}

Overall, 31 patients (3.2\%) (14 males, 17 females) with a mean age of $44.2 \pm 16.7$ years had musculoskeletal tuberculosis. The mean duration of disease was $12.9 \pm 5.5$ months. Of the 31 patients, six (19.4\%) had concomitant pulmonary TB. One of the patients (3.2\%) was in paediatrics age group, and two of them (6.4\%) were in geriatric group. The most affected area was the vertebra. The most common complaint of the patients was back pain and seen in 22 patients (70.9\%).

\section{Conclusions}

The physicians should be suspicious about the diagnosis of musculoskeletal TB infection. If the diagnosis and treatment is delayed, spinal damage and other consequences might be incurable.

\section{Introduction}

Tuberculosis (TB) is a chronic infectious disease mainly caused by Mycobacterium tuberculosis. TB is a public health problem, especially in underdeveloped or developing countries, and remains a major cause of morbidity and mortality (1). As reported by World Health Organization (WHO), the estimated global incidence of TB cases was 10.0 million in 2018 (2). In Turkey, as a developing country, the TB incidence (the number of new TB cases per 100,000 populations per year) decreased annually, from 29.8 in 2005 to 14.4 in $2018(3,4)$.

More than $80 \%$ of TB cases are pulmonary TB. However, several manifestations of extra-pulmonary TB have been reported, including musculoskeletal system TB (5). Although pulmonary TB has been 
extensively covered in the literature (6-8), publications on musculoskeletal TB are relatively limited, and have been reported mostly in case reports (9-11). In addition, the diagnosis of musculoskeletal system TB can be challenging, particularly for the physicians who are not familiar, because TB can present in atypical locations and mimic tumoral lesions (12). Therefore, a biopsy should be taken to confirm the diagnosis.

Histopathologically, a granulomatous inflammatory reaction that occurs against mycobacterial species is composed of epithelioid histiocytes, giant cells, lymphocytes, and some of them have centrally located caseous necrosis. The granuloma can not only occur against TB disease, but also against fungi as an infectious agent, sarcoidosis as an immunological reaction, or against a foreign body. Histochemically, Mycobacterium tuberculosis bacilli can be demonstrated inside the granuloma, mainly necrotic areas with Erlich-Ziehl-Neelsen (EZN) stain to confirm the diagnosis. Also, the diagnosis can be made by culture and amplification of Mycobacterium tuberculosis DNA using the polymerase chain reaction (PCR) method. The diagnosis of musculoskeletal TB is usually tricky and could be delayed. A positive skin tuberculin test or abnormal chest radiograph will support the diagnosis though it is not excluded by negative results (13).

Respecting immunosuppression, the incidence of musculoskeletal TB and all other extra-pulmonary TB infections depend on the grade of weakening cellular immunity. People with human immunodeficiency virus/acquired immunodeficiency syndrome (HIV/AIDS) and latent TB are also far more likely to progress active disease, with a nearly $10 \%$ risk of developing active disease each year, in comparison with a lifelong reactivation risk of about $5 \%$ in an HIV/AIDS-negative population. As impairment of the immune system progresses, patients living with HIV/AIDS are more likely to develop extra-pulmonary TB, just like musculoskeletal TB (14).

Keeping in mind the increased prevalence of TB in recent years (15), we deem it important to review musculoskeletal involvement of TB. Accordingly, the objective of this study was to elaborate musculoskeletal system TB as a single-center experience.

\section{Materials And Methods}

This retrospective study was conducted as a descriptive single-center experience. Patients who were followed up in xxx Tuberculosis Dispensary between 2004 and 2020 were screened from the dispensary records. Among them, cases with musculoskeletal system involvement were noted. All patients were diagnosed with tuberculosis according to the histopathologic examinations by a pathologist. The biopsy samples were evaluated grossly and processed using the routine histopathologic technique (fixation and paraffin embedding) and then stained with Haematoxylin-Eosin.

Definite diagnosis of musculoskeletal TB was made when either (I) joint aspiration or tissue biopsy/fineneedle aspiration cytology (FNAC) revealed acid-fast bacilli (AFB) detection by smear examination, (II) culture of the synovial fluid (or tissue aspirated) grew Mycobacterium tuberculosis, or (III) histopathology/FNAC revealed granulomatous lesions with or without caseation with AFB positivity. 
Probable TB was considered if there was no explicit evidence of AFB or granuloma, but clinical, radiological, and serological evidence suggested TB and the patient responded to empirical antitubercular therapy. Other tests include tuberculin skin sensitivity (PPD) test and TB interferon $\mathrm{y}$-assays. Radiology included X-ray, magnetic resonance imaging (MRI), or computed tomography (CT) of the organ involved. CT-guided biopsy or fine-needle aspiration and arthroscopic biopsies were also performed in some cases.

The clinical and demographic characteristics, including age, gender, involvement location and duration of illness, presenting complaint, local examination findings, treatment outcome were retrieved and analyzed from the case files. TB is a notifiable disease, and data are recorded prospectively by our specialist TB nurses. Patients with missing data in terms of diagnosis, clinical follow-up, or treatment, and patients transferred to another tuberculosis center were excluded.

Typical treatment of all cases included the use of a four-drug regimen: isoniazid, rifampicin, ethambutol, and pyrazinamide for the initiative phase of two months, followed by 4-10 months of continuation phase with isoniazid and rifampicin. If the case was a recurrence TB, streptomycin applied to the patients intravenously (IV) is added to the treatment regimen for first two months. The drug doses were adjusted according to the weight of the patients. Drug susceptibility testing for the first-line drugs was conducted for patients who did not respond to treatment, and regimens were changed based on the susceptibility results or strong clinical suspicion of unresponsiveness.

Statistical analyses were performed using SPSS Statistics version 17.0 (IBM). The normality of data analyzed by using Kolmogorov-Smirnov. The descriptive statistics were reported as mean \pm standard deviation, medians, and ranges (min-max).

\section{Results}

A total of 949 patients were followed up due to tuberculosis in our center between 2004 and 2020 . Overall, 31 (3.2\%) patients (14 males, 17 females) with a mean age of $44.2 \pm 16.7$ years (ranges: 3 to 75 years) had musculoskeletal tuberculosis. Demographic features are summarized in Table 1.

\section{Table 1. Demographic Features}




\begin{tabular}{|c|c|}
\hline Variables & Results \\
\hline Age (years)* & $44.2 \pm 16.7 \quad 49(3-75)$ \\
\hline Gender $n,(\%)$ & \\
\hline 1. Male & $\begin{array}{l}14(45.2) \\
17(54.8)\end{array}$ \\
\hline 2. Female & \\
\hline Age group (years) n, (\%) & \\
\hline - $\quad$ Pediatric $(<18)$ & $1(3.2)$ \\
\hline - Adult (18-64) & $28(90.3)$ \\
\hline - Geriatric $(\geq 65)$ & $2(6.5)$ \\
\hline Race n, (\%) & \\
\hline & $29(93.5)$ \\
\hline 15. Turkish & $1(3.2)$ \\
\hline 3. Syria & \\
\hline Occupation $\mathrm{n},(\%)$ & \\
\hline Housewife & $12(38.7)$ \\
\hline Butcher & $4(12.9)$ \\
\hline Student & $4(12.9)$ \\
\hline Retired & $2(6.5)$ \\
\hline Worker & $2(6.5)$ \\
\hline Farmer & $1(3.2)$ \\
\hline Cooker & $1(3.2)$ \\
\hline Craftsman & $1(3.2)$ \\
\hline $\begin{array}{l}\text { Family history of TB } n,(\%) \\
\end{array}$ & $6(19.4)$ \\
\hline Concomitant Pulmonary TB n, (\%) & $6(19.4)$ \\
\hline
\end{tabular}

$*$ mean \pm S.D and median (minimum-maximum)

TB: Tuberculosis

Clinical presentations of the cases are given in Table 2. Back pain $(n=22,70.9 \%)$ and shoulder pain $(n=$ $2,6.5 \%)$ were the most seen symptoms, and thoracic or lumbar vertebra was the most involved location (74.2\%). The example images of a shoulder TB are also shown in Figs. 1 and 2. Most of the cases $(58.1 \%)$ were diagnosed in tertiary hospitals. The mean duration of treatment was $12.9 \pm 5.5$ months (range, 1.3 week to 27.7 months). Two of the patients had a recurrent illness and were treated with the HRZE + S (isoniazid, rifampicin, ethambutol, and pyrazinamide + IV streptomycin) regimen. 
Table 2

Clinical Presentation $\mathrm{n},(\%)$

\begin{tabular}{|ll|}
\hline Variable & Data \\
\hline Symptoms & $22(70.9)$ \\
- Back pain & $1(3.2)$ \\
- Mass on back and neck & $1(3.2)$ \\
- Knee pain & $1(3.2)$ \\
- Wrist swelling & $1(3.2)$ \\
- Chest pain & $1(3.2)$ \\
- Hand pain & $2(6.5)$ \\
- Shoulder pain & $1(3.2)$ \\
- Abdominal/hip pain & \\
\hline Location & $23(74.2)$ \\
- Vertebra & $2(6.5)$ \\
- Hand & $1(3.2)$ \\
- Knee joint & $1(3.2)$ \\
- Costa & $1(3.2)$ \\
- Shoulder & $1(3.2)$ \\
- Patella & $2(6.5)$ \\
- Psoas muscle & $1(3.2)$ \\
\hline Vertebra Location & $10(32.3)$ \\
- Thoracic & $12(38.7)$ \\
- Lumbar & \\
- Sacrococcygeal & \\
\hline
\end{tabular}

*mean \pm S.D \& median (percentile 25-percentile 75)

TB: Tuberculosis

HRZE: isoniazid + rifampicin + ethambutol + pyrazinamide

HRZE + S: isoniazid + rifampicin + ethambutol + pyrazinamide + streptomycin 


\begin{tabular}{|ll|}
\hline Variable & Data \\
\hline Center of Diagnosis & $13(41.9)$ \\
- Secondary & $18(58.1)$ \\
- Tertiary & $12.9 \pm 5.5 \& 12.0(1.3-27.7)$ \\
\hline Treatment duration (months)* & \\
\hline Outcome & $26(83.8)$ \\
\hline - Treatment completed & $3(9.6)$ \\
\hline - Treatment is going on & $1(3.2)$ \\
\hline - Exitus & $1(3.2)$ \\
- Leaved treatment & $29(93.5)$ \\
\hline Primer TB & $2(6.5)$ \\
\hline Recurrence TB & $29(93.5)$ \\
\hline Treatment Regimen & $2(6.5)$ \\
\hline - HRZE & \\
\hline - HRZE + S & \\
\hline *mean \pm S.D \& median (percentile 25-percentile 75) \\
\hline TB: Tuberculosis & \\
\hline HRZE: isoniazid + rifampicin + ethambutol + pyrazinamide \\
\hline HRZE + S: isoniazid + rifampicin + ethambutol + pyrazinamide + streptomycin \\
\hline
\end{tabular}

\section{Bursit Case}

The example images are from a 49-year-old man who presented with fatigue, left shoulder pain, and restriction in shoulder movements (Fig. 3). Laboratory investigations yielded increased levels of Creactive protein and erythrocyte sedimentation rate. MRI showed joint effusion, synovial hypertrophy, bursitis, rice bodies, and cortical erosions. The pathological examination was consistent with lymphohistiocytic inflammatory response, including giant cells and caseified necrosis in adipose and connective tissue (i.e., granulomatous inflammatory response). The patient was received a 12 monthtreatment (2 months HRZE + 10 months HR).

\section{Discussion}

The majority of extra-pulmonary TB cases involve the pleura, musculoskeletal and lymphatic systems. Most cases verified to have a previous pulmonary TB origin (16). TB bacilli spread over the pleural cavity, 
starting from a pulmonary infection focus on that occasion, immigrates through the blood vessels or lymphatics to other organs developing extrapulmonary TB.

The distribution of age and site of musculoskeletal TB has been previously studied. Overall, of all TB cases, $1 \%-4 \%$ showed musculoskeletal involvement. In a retrospective and observational study conducted in a tertiary area with the highest prevalence of TB worldwide, three peaks in the first, third, and sixth decade of life were found (17). However, some reports highlighted a bimodal age distribution (18). In a nine-year single-center experience, the rate of musculoskeletal TB was found as $4 \%$ (19). In our study, the rate of musculoskeletal system TB was $3.2 \%$, which was compatible with the previous reports. On the other hand, the mean age was 44 years, and $90 \%$ of all cases were adult patients. We did not find an age peak in childhood or in the advanced age group. Musculoskeletal TB is distributed almost equally among males and females in our sample group.

In the literature, most of the TB cases present with spine involvement. More than $10 \%$ of patients with extra-pulmonary TB have skeletal involvement (20). The most common form of skeletal TB is the Pott's disease, comprising approximately half of musculoskeletal TB cases, and followed by TB arthritis and extra-spinal TB osteomyelitis (21). In the retrospective study done by Michael et al. (17), 78\% of the cases had spine TB while the remaining $21.6 \%$ had extra-spinal diseases, comprising hip, knee, foot/ankle, shoulder, elbow, wrist, and others. The thoracic region of the vertebral column is commonly involved (20). Likewise, in our study, the prevalent anatomically affected location was the spine (23 of 31 patients; $75 \%$ ) as well as hands, knee joint, costa, shoulder, patella, and psoas muscle. Only two cases in this study were involved with psoas muscle. These two cases had both concomitant pulmonary TB. The psoas muscle is a retroperitoneal muscle that originates from the lateral borders of T12 to L5 vertebrae and ends as a tendon that inserts into the lesser trochanter. The primary TB of iliopsoas compartment abscess with occult cause rarely encountered in the clinical practice and is generally idiopathic.

The joints' TB infection comes after hematogenous spread or direct invasion from neighboring tissue of TB osteomyelitis. Mostly monoarticular joints are involved such as the knee and hip.

Oligoarticular/polyarticular patterns are very rare, ranging from $5-15 \%$ of cases, sometimes with small joint involvement, and ordinary in immunosuppressed patients (22). A patient in our series (male, 38-yearold, butcher), TB was detected in the biopsy performed a long time after the local infectious swelling developed with a knife sticking in the left hand at the workplace.

The delays in the diagnosis of musculoskeletal TB have been sufficiently presented (13). This may be due to the patients' uncertain histories, perhaps complicated by inaccurate stories of irrelevant trauma, and lack of presence of a concomitant pulmonary involvement. In the current study, complaints of $70.9 \%$ of cases have been started as back pain. Therefore, these patients have been investigated in neurosurgery, orthopedic and traumatology, or algology clinics for a long time. The patients usually presented with nonspecific symptoms such as back pain, mass on back/neck, knee pain, wrist swelling, chest pain, shoulder pain, hand pain, or abdominal pain. 
There were some limitations to our work. Primarily, because of the retrospective nature of our study, it was not possible to obtain detailed information of every patient. Furthermore, because the patient registry systems of dispensaries and hospitals are not integrated, monitoring and clinical information that are not recorded in the ledger could not be accessed.

\section{Conclusions}

To sum up, since it is a very rare disease, surgeons and clinicians should be very skeptical about the diagnosis of musculoskeletal TB infection. On the assumption that the diagnosis and treatment is delayed, spinal damage in addition to the bones and joints of the patients may be an inevitable consequence. Consequently, musculoskeletal TB needs a complex approach and cooperation.

\section{List Of Abbreviations}

AFB: acid-fast bacilli

CT: computed tomography

EZN: Erlich-Ziehl-Neelsen

FNAC: fine-needle aspiration cytology

HIV/AIDS: human immunodeficiency virus/acquired immunodeficiency syndrome

HRZE+S: isoniazid, rifampicin, ethambutol, and pyrazinamide + streptomycin

IV: intravenously

MRI: magnetic resonance imaging

PCR: polymerase chain reaction

PPD: tuberculin skin sensitivity

TB: tuberculosis

WHO: World Health Organization

\section{Declarations}

\section{Ethics approval and consent to participate}

The current study protocol was approved by the Local Ethics Committee of Adana City Training and Research Hospital (decision number: 321/2018). This study was exempted from informed consent form 
by this ethics committee, since it was a retrospective study. All authors confirmed that all methods were performed in accordance with the relevant guidelines and regulations.

\section{Consent for publication}

The written consents of publication of the typical MRI and microscopic images were obtained from patients.

\section{Availability of data and materials}

The datasets used in this study are available from the corresponding author on reasonable request.

\section{Competing interests}

The authors declared no conflicts of interest with respect to the authorship and/or publication of this article.

\section{Funding}

The authors received no financial support for the research and/or authorship of this article.

\section{Authors' contributions}

MO, HGO conceived and designed the study; MO collected clinical data and analysed the data, HGO prepared figures 1 and 2, MO, HGO wrote the manuscript. All authors read and approved the final manuscript.

collecting clinical data: MO, HGO; analysed the data: MO; writing: MO,HGO. All authors read and approved the final manuscript.

\section{Acknowledgements}

Not applicable.

\section{References}

1. Zaman K. Tuberculosis: a global health problem. Journal of health, population, and nutrition. 2010;28(2):111-3.

2. Harding E. WHO global progress report on tuberculosis elimination. The Lancet Respiratory medicine. 2020;8(1):19.

3. Bora Basara B, Soytutan Caglar I, Aygun A, Ozdemir T. Health Statistics Yearbook 2018. Ankara, Turkey: Republic of Turkey, Ministry of Health, General Directorate of Health Information Systems. 2019. 
4. Ozdemir M. Osmaniye İli Verem Savaş Dispanserliğine Başvuran Hastaların Retrospektif Değerlendirilmesi. Turkish Journal of Family Medicine and Primary Care. 2020;14(2):252-8.

5. Leonard MK, Blumberg HM. Musculoskeletal Tuberculosis. Microbiology spectrum. 2017;5(2).

6. Loddenkemper R, Lipman M, Zumla A. Clinical Aspects of Adult Tuberculosis. Cold Spring Harbor perspectives in medicine. 2015;6(1):a017848.

7. Furlow B. Tuberculosis: a review and update. Radiologic technology. 2010;82(1):33-52.

8. Campbell IA, Bah-Sow 0. Pulmonary tuberculosis: diagnosis and treatment. BMJ (Clinical research ed). 2006;332(7551):1194-7.

9. Kandwal P, G V, Jayaswal A. Management of Tuberculous Infection of the Spine. Asian spine journal. 2016;10(4):792-800.

10. Hogan JI, Hurtado RM, Nelson SB. Mycobacterial Musculoskeletal Infections. Infectious disease clinics of North America. 2017;31(2):369-82.

11. Hogan JI, Hurtado RM, Nelson SB. Mycobacterial Musculoskeletal Infections. Thoracic surgery clinics. 2019;29(1):85-94.

12. Wagh Y, Reddy R, Gundavda M, Agarwal M, Agashe VM, Bajwa S. Tuberculosis (T.B.) masquerading as tumor. An 8-year study on 25 cases of long bone tuberculosis presenting as tumors. Sicot-j. 2020;6:14.

13. Davidson PT, Horowitz I. Skeletal tuberculosis. A review with patient presentations and discussion. The American journal of medicine. 1970;48(1):77-84.

14. Sharma SK, Mohan A, Kadhiravan T. HIV-TB co-infection: epidemiology, diagnosis \& management. The Indian journal of medical research. 2005;121(4):550-67.

15. Coskun O, Safaz I, Gul CH, Ozgul A, Eyigun CP. Heterotopic ossification in a patient with tuberculous meningoencephalitis. Internal medicine (Tokyo, Japan). 2008;47(24):2195-6.

16. Lin JN, Lai CH, Chen YH, Lee SS, Tsai SS, Huang CK, et al. Risk factors for extra-pulmonary tuberculosis compared to pulmonary tuberculosis. The international journal of tuberculosis and lung disease: the official journal of the International Union against Tuberculosis and Lung Disease. 2009;13(5):620-5.

17. Held MFG, Hoppe S, Laubscher M, Mears S, Dix-Peek S, Zar HJ, et al. Epidemiology of Musculoskeletal Tuberculosis in an Area with High Disease Prevalence. Asian spine journal. 2017;11(3):405-11.

18. Colmenero JD, Jiménez-Mejías ME, Reguera JM, Palomino-Nicás J, Ruiz-Mesa JD, Márquez-Rivas J, et al. Tuberculous vertebral osteomyelitis in the new millennium: still a diagnostic and therapeutic challenge. European journal of clinical microbiology \& infectious diseases: official publication of the European Society of Clinical Microbiology. 2004;23(6):477-83.

19. Pazarlı AC, Ekiz T, Abakay MA. Clinical presentation of tuberculosis: a nine-year single-center experience. The European Research Journal. 2018;4(3):211-4. 
20. Garg RK, Somvanshi DS. Spinal tuberculosis: a review. The journal of spinal cord medicine. 2011;34(5):440-54.

21. Shah BA, Splain S. Multifocal osteoarticular tuberculosis. Orthopedics. 2005;28(3):329-32.

22. Vohra R, Kang HS, Dogra S, Saggar RR, Sharma R. Tuberculous osteomyelitis. The Journal of bone and joint surgery British volume. 1997;79(4):562-6.

\section{Figures}

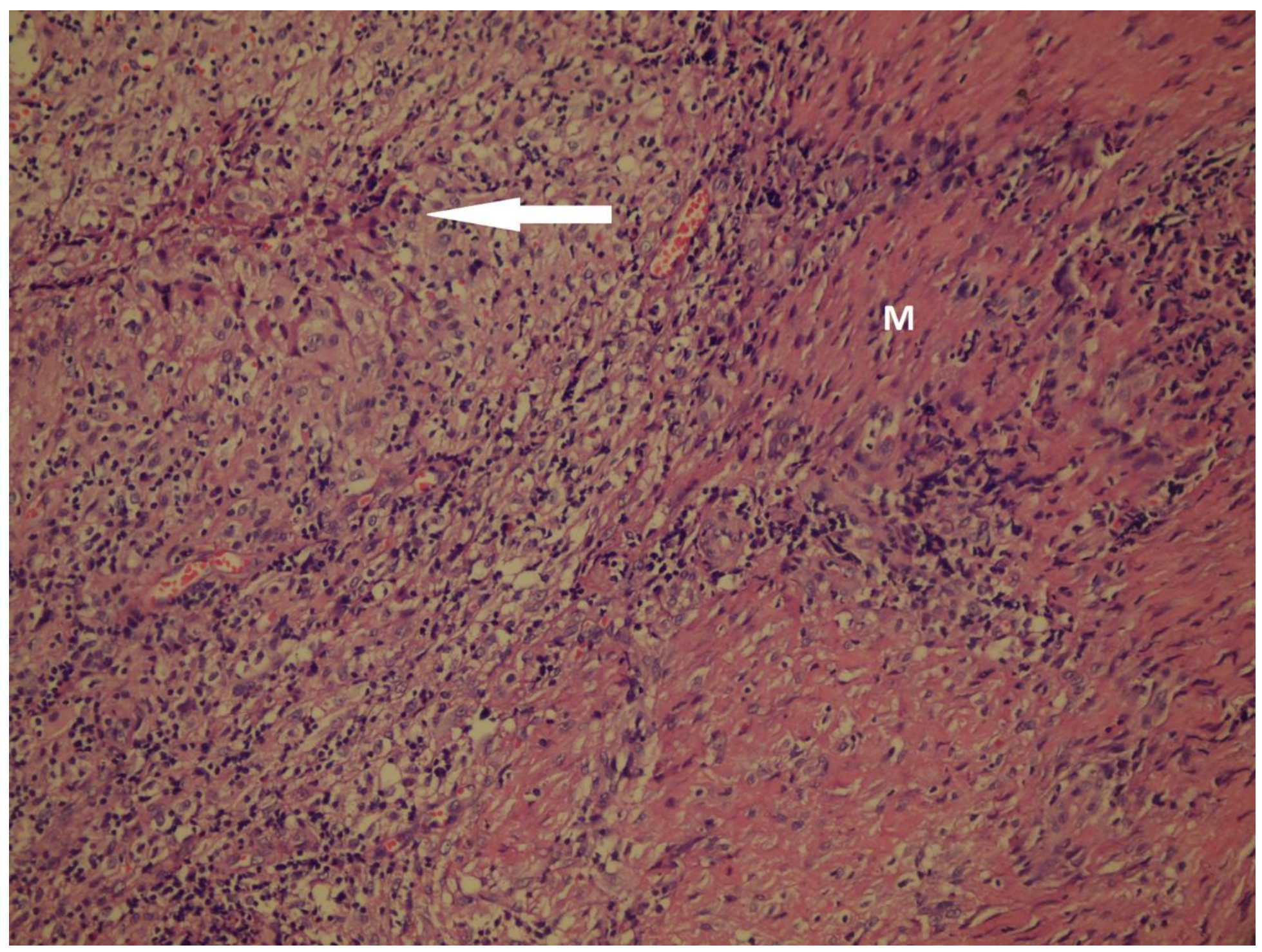

\section{Figure 1}

Granulomatosis inflammatory reaction against M. tuberculosis with caseous necrosis (white arrow) seen in muscle tissue $(M)(\times 200, H \& E)$ 


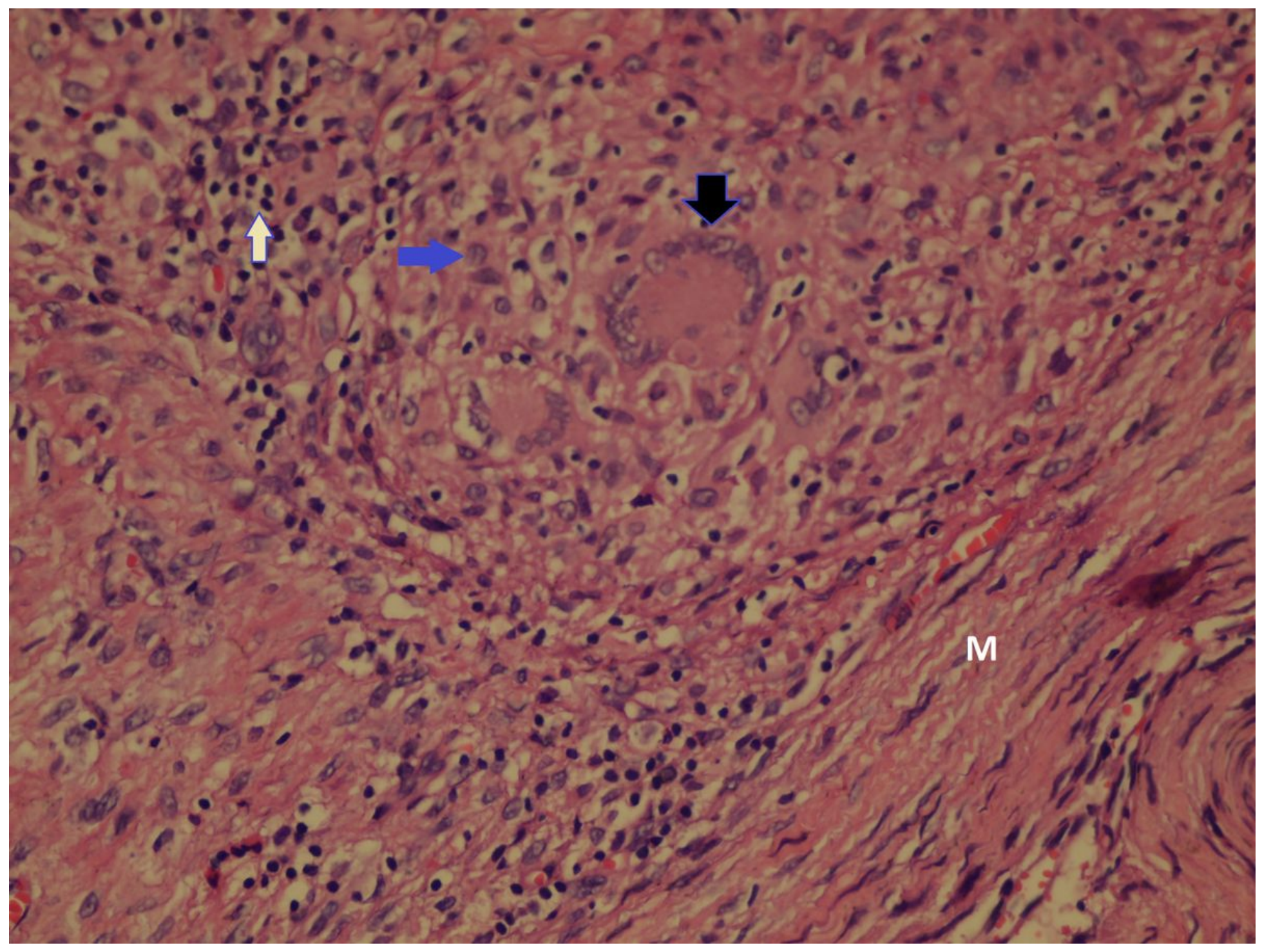

Figure 2

Epithelioid histiocytes (blue arrow), lymphocytes (yellow arrow) and Langhans giant cells (black arrow) forms granuloma in muscle tissue (M) (x400, H\&E) 


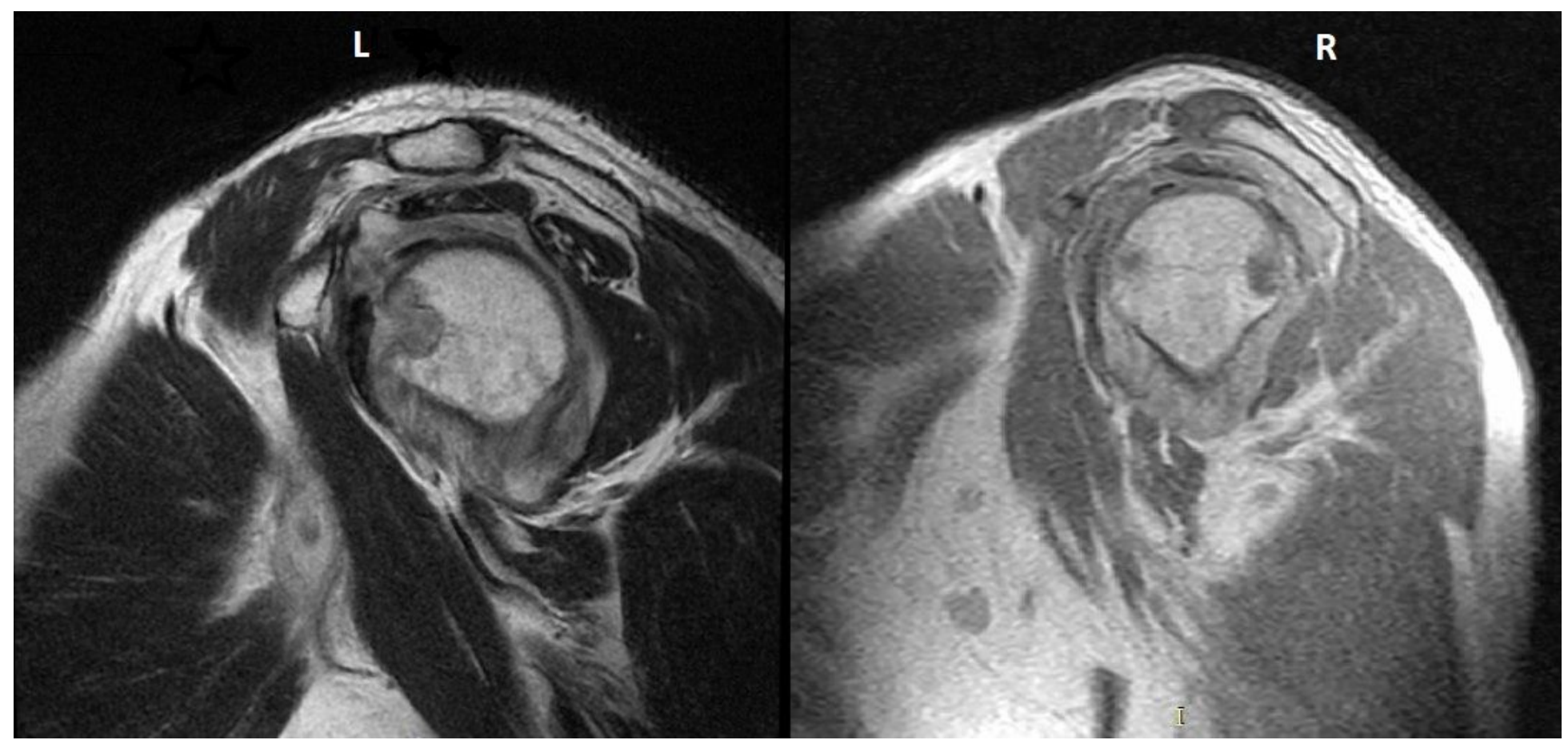

Figure 3

Preoperative (left) and postoperative (right) Magnetic Rezonans Imaging (sagittal plane T1) of bursitis case 